Europhys. Lett., 75 (5), pp. 716-722 (2006)

DOI: $10.1209 / \mathrm{epl} / \mathrm{i} 2006-10190-9$

\title{
Monte Carlo study of the evaporation/condensation transition of Ising droplets
}

\author{
A. NußBaumer ${ }^{1}$, E. Bittner $^{1}$, T. Neuhaus ${ }^{2}$ and W. Janke ${ }^{1}$ \\ 1 Institut für Theoretische Physik and Centre for Theoretical Sciences (NTZ) \\ Universität Leipzig - Augustusplatz 10/11, D-04109 Leipzig, Germany \\ 2 John von Neumann-Institut für Computing, Forschungszentrum Jülich \\ D-52425 Jülich, Germany
}

received 7 May 2006; accepted in final form 18 July 2006

published online 10 August 2006

PACS. 05.70.Fh - Phase transitions: general studies.

PACS. 02.70.Uu - Applications of Monte Carlo methods.

PACS. 75.10. Hk - Classical spin models.

\begin{abstract}
In recent analytical work, Biskup et al. (Europhys. Lett., 60 (2002) 21) studied the behaviour of $d$-dimensional finite-volume liquid-vapour systems at a fixed excess $\delta N$ of particles above the ambient gas density. By identifying a dimensionless parameter $\Delta(\delta N)$ and a universal constant $\Delta_{\mathrm{c}}(d)$, they showed in the limit of large system sizes that for $\Delta<\Delta_{\mathrm{c}}$ the excess is absorbed in the background ("evaporated" system), while for $\Delta>\Delta_{\mathrm{c}}$ a droplet of the dense phase occurs ("condensed" system). Also the fraction $\lambda_{\Delta}$ of excess particles forming the droplet is given explicitly. Furthermore, they argue that the same is true for solid-gas systems. By making use of the well-known equivalence of the lattice-gas picture with the spin- $(1 / 2)$ Ising model, we performed Monte Carlo simulations of the Ising model with nearest-neighbour couplings on a square lattice with periodic boundary conditions at fixed magnetisation, corresponding to a fixed particles excess. To test the applicability of the analytical results to much smaller, practically accessible system sizes, we measured the largest minority droplet, corresponding to the solid phase, at various system sizes $(L=40, \ldots, 640)$. Using analytic values for the spontaneous magnetisation $m_{0}$, the susceptibility $\chi$ and the Wulff interfacial free energy density $\tau_{\mathrm{W}}$ for the infinite system, we were able to determine $\lambda_{\Delta}$ numerically in very good agreement with the theoretical prediction.
\end{abstract}

Introduction. - The formation and dissolution of equilibrium droplets at a first-order phase transition is one of the longstanding problems in statistical mechanics [1]. Quantities of particular interest are the size and free energy of a "critical droplet" that needs to be formed before the decay of the metastable state via homogeneous nucleation can start. For large but finite systems, this is signalised by a cusp in the probability density of the order parameter $\phi$ towards the phase-coexistence region as depicted in figs. 1 and 2 for the example of the twodimensional (2D) Ising model, where $\phi=m$ is the magnetisation. This "transition point" separates an "evaporated" phase with many very small bubbles of the "wrong" phase around the peak at $\phi_{0}$ from the "condensed phase" phase, in which a large droplet has formed; for configuration snapshots see fig. 3. The droplet eventually grows further towards $\phi=0$ until it 


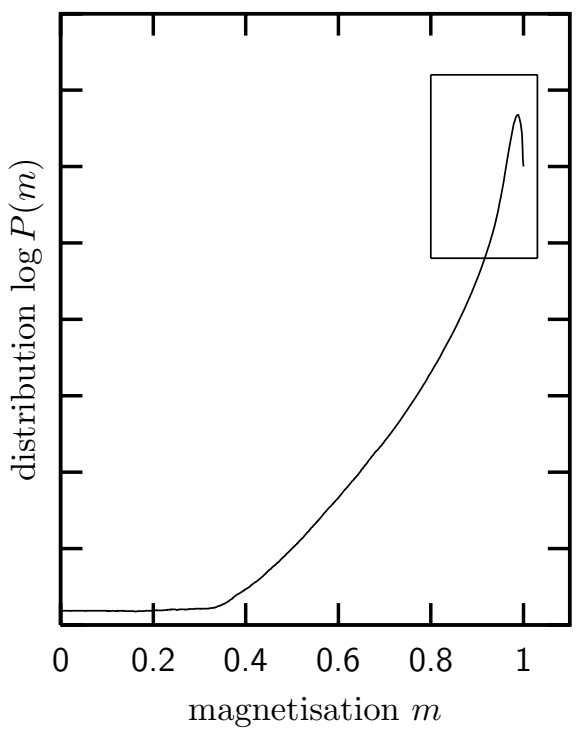

Fig. 1

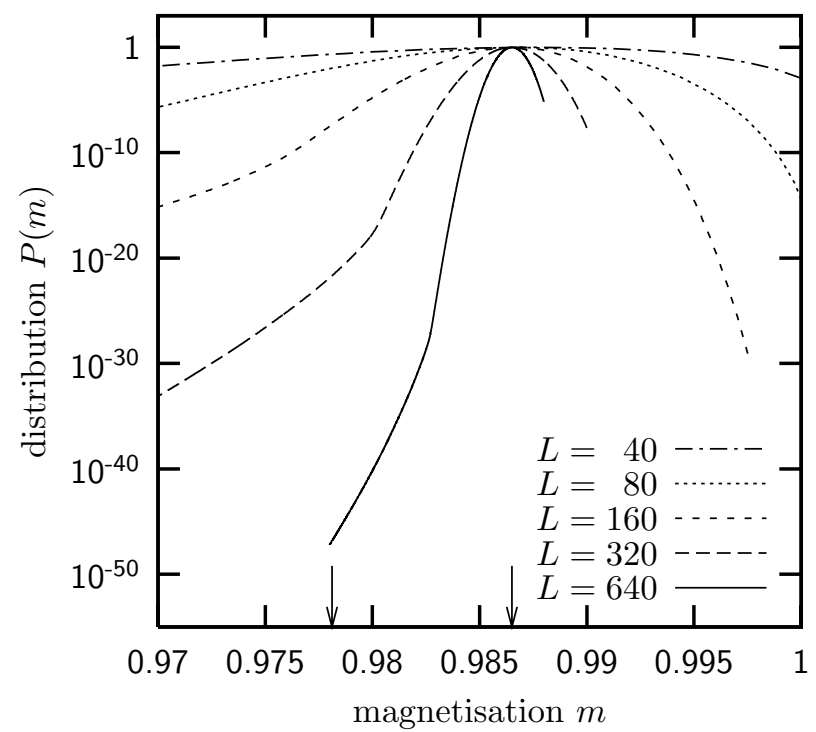

Fig. 2

Fig. 1 - Schematic plot of the probability density $\log P(m)$ of the magnetisation in logarithmic form. The marked box indicates the position of the cut-out displayed in fig. 2 .

Fig. 2 - Probability density of the magnetisation for the two-dimensional Ising model around its right peak for different system sizes $L$ at the temperature $T=1.5$. The cusp indicates the evaporation/condensation transition region. On the right side of the cusp (evaporated system) a Gaussian peak is clearly visible, while on the left side (condensed system) a stretched exponential behaviour can be seen. The two arrows on the $x$-axis indicate for $L=640$ the range of data points shown in fig. 5 .

percolates the finite system in another "droplet-strip" transition. The latter transition is indicated in the 2D Ising model by the cusp at the beginning of the flat two-phase region (see fig. 1).

Building on the seminal work by Fisher [1] on the droplet picture, early numerical studies of the evaporation/condensation transition by Binder, Kalos and Furukawa $[2,3]$ date back to the beginning of the 1980s. Recently, this problem has been taken up again by Hager and one of the authors [4] who discussed it with emphasis on possible Gibbs-Thomson and Tolman corrections. This stimulated further new theoretical [5-7] and numerical [8] work. Here, we follow the exposition of Biskup et al. $[5,6]$, who present their results both in a phenomenological
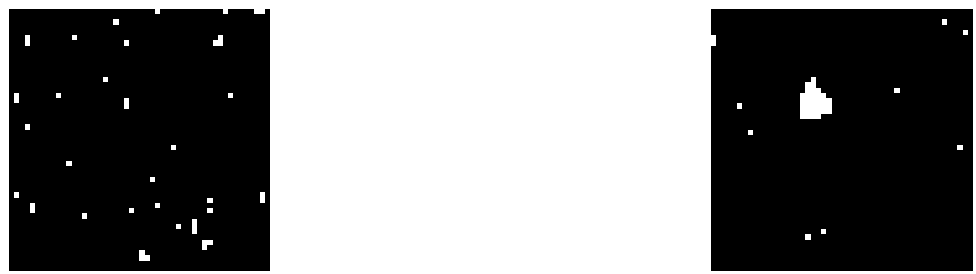

Fig. 3 - Two snapshots of a $L=50$ system at the same value of the magnetisation. Left: evaporated system, a large number of very small bubbles exist (1 to 3 spins). Right: condensed system, a single large droplet that has absorbed nearly all small bubbles. 
liquid-vapour (or solid-gas) picture and also explicitly in terms of the simple Ising (lattice-gas) model. The distinguishing feature of their work is the formulation of a proper equilibrium theory which does not need to explicitly involve correction effects à la Gibbs-Thomson or Tolman [9]. We consider this feature as one of the main merits of their formulation which can be shown to be equivalent (at least in leading order) to the earlier less rigorous treatment in [4].

The price one has to pay, however, is a rather intricate rescaling of the original problem which requires in numerical work great care with details. To set the theoretical grounds for our Monte Carlo simulation study and, in particular, to develop intuition for the final representation of our results in fig. 5 below, we therefore start first with a brief summary of the Biskup et al. $[5,6]$ theory. In order to so, we restrict ourselves to the special case of the 2D Ising model with a Hamiltonian

$$
\mathcal{H}=-J \sum_{\langle i, j\rangle} \sigma_{i} \sigma_{j}
$$

where $\sigma_{i}= \pm 1$ and $\langle i, j\rangle$ denotes a nearest-neighbour pair. If an up-spin $\left(\sigma_{i}=1\right)$ is treated as a particle and a down-spin $\left(\sigma_{i}=-1\right)$ as a vacancy, the system can be interpreted as a lattice gas of atoms.

Theory. - Consider the 2D Ising model (1) in the low-temperature phase at an inverse temperature $\beta \equiv J / k_{\mathrm{B}} T>\beta_{\mathrm{c}}=\ln (1+\sqrt{2}) / 2$ and denote the infinite-volume equilibrium magnetisation per site by $m_{0}=m_{0}(\beta)>0$, i.e., assume that the system is in the phase with positive magnetisation. For a system of finite volume $V=L \times L$, we now ask how fluctuations can drive the nucleation of the "wrong" phase with negative magnetisation $-m_{0}$. If the "wrong" phase occupies a total volume $v_{L}$, the total magnetisation of the system $M=\sum_{i} \sigma_{i}$ can be expressed as $M=-m_{0} v_{L}+m_{0}\left(V-v_{L}\right)$. Rearranging this expression for the difference in the magnetisation yields

$$
\delta M=M-V m_{0}=-2 v_{L} m_{0} .
$$

Next, to answer the question as to how the nucleation of the "wrong" phase proceeds, two contributions to the free energy can be easily identified. First, there can be local fluctuations leading to many small nucleation bubbles. If the total excess in the magnetisation (compared to $M_{0}=m_{0} V$ ) of $\delta M=M-M_{0}$ goes into these fluctuations, then the probability to find $\delta M$ can be expressed in terms of a Gaussian distribution as

$$
\exp \left[-\frac{(\delta M)^{2}}{2 V \chi}\right]=\exp \left[-\frac{\left(2 m_{0} v_{L}\right)^{2}}{2 V \chi}\right],
$$

where $\chi=\chi(\beta)=\beta V\left[\left\langle m^{2}\right\rangle-\langle m\rangle^{2}\right]$ is the susceptibility in the thermodynamic limit.

In the second extreme case, the total excess is taken up by a single large droplet of volume $v_{L}$ (cf. fig. 4). The cost to form it depends on the interface of the droplet, which is given in two dimensions [10] as

$$
\exp \left[-\tau_{\mathrm{W}} \sqrt{v_{L}}\right],
$$

where $\tau_{\mathrm{W}}=\tau_{\mathrm{W}}(\beta)$ is the interfacial free energy per unit volume of an ideally shaped droplet, also known as the free energy of a droplet of Wulff shape [11].

Comparing the exponents of the two limiting cases in eqs. (3) and (4) gives

$$
\Delta=\frac{\left(2 m_{0} v_{L}\right)^{2} /(2 V \chi)}{\tau_{\mathrm{W}} \sqrt{v_{L}}}=2 \frac{m_{0}^{2}}{\chi \tau_{\mathrm{W}}} \frac{v_{L}^{3 / 2}}{V} .
$$

With $\Delta \stackrel{!}{=} 1$ (the free energy contributions of the droplet and the fluctuations are of equal size) and eq. (2), the difference in the magnetisation (which is a negative quantity) can be 


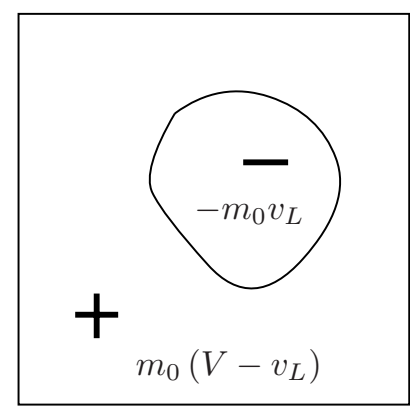

Fig. 4 - Ising system of size $V$ with a minority droplet of volume $v_{L}$ of negative spins surrounded by positive spins with a volume $\left(V-v_{L}\right)$, shown in the extreme case where the total excess in magnetisation is concentrated in the droplet, i.e. $v_{d}=v_{L}$.

expressed as

$$
-\delta M=\theta V^{2 / 3} \quad \text { with } \quad \theta=\left(\frac{2 \chi \tau_{\mathrm{W}}}{\sqrt{2 m_{0}}}\right)^{2 / 3} .
$$

Under the assumption that the total excess goes into either one of the two excitations, this means that the droplet mechanism dominates when $-\delta M \gg \theta V^{2 / 3}$, while the fluctuation mechanism dominates for $-\delta M \ll \theta V^{2 / 3}$. A priori one would suspect, however, that both types of excitations can coexist, with the excess volume $v_{L}$ split up between the local fluctuations and the droplet. Biskup et al. [5,6] studied the crossover region $-\delta M \propto v_{L} \propto \theta V^{2 / 3}$. By isoperimetric reasoning, they proved that in this range no droplets of intermediate size can exist [6]. Hence, there is at most a single large droplet of size $v_{\mathrm{d}}<v_{L}$ with costs $\exp \left[-\tau_{\mathrm{W}} \sqrt{v_{d}}\right]$ that absorbs $\delta M_{\mathrm{d}}=-2 v_{\mathrm{d}} m_{0}$ of the complete excess of the magnetisation $\delta M$, while the rest $v_{L}-v_{\mathrm{d}}$ goes into the fluctuations of the background with costs exp $\left[-\left(\delta M-\delta M_{d}\right)^{2} /(2 V \chi)\right]$. In the limit of large system sizes, this justifies for the probability of the excess in magnetisation the ansatz

$$
\exp \left[-\tau_{\mathrm{W}} \sqrt{v_{\mathrm{d}}}-\frac{\left(\delta M-\delta M_{\mathrm{d}}\right)^{2}}{2 V \chi}\right]=\exp \left[-\tau_{\mathrm{W}} \sqrt{\frac{-\delta M}{2 m_{0}}} \Phi_{\Delta}(\lambda)\right], \quad \Phi_{\Delta}(\lambda)=\left[\sqrt{\lambda}+\Delta(1-\lambda)^{2}\right],
$$

where $\lambda=\delta M_{\mathrm{d}} / \delta M$ is the fraction taken up by the droplet and $\Delta$ is defined in eq. (5). Taking into account that $\tau_{\mathrm{W}} \sqrt{-\delta M / 2 m_{0}}$ and $\Delta$ are constants, the fraction of excess that is most probable is obtained by minimising $\Phi_{\Delta}(\lambda)$. As a function of $\Delta$, the solution $\lambda_{\Delta}$ behaves like at a first-order phase transition. For values $\Delta<\Delta_{\text {c }}$ the global minimum of $\Phi_{\Delta}(\lambda)$ is reached for $\lambda=0$, while for $\Delta>\Delta_{\mathrm{c}}$ it is located at a nontrivial value $\lambda_{\Delta}>0$. At the transition point $\Delta=$ $\Delta_{\mathrm{c}}$, the solution jumps to a value $\lambda_{\mathrm{c}}>0$. In two dimensions, one obtains $\Delta_{\mathrm{c}}=(1 / 2)(3 / 2)^{3 / 2} \approx$ 0.92 and $\lambda_{c}=2 / 3$. The solid line in fig. 5 below shows the graph of $\lambda_{\Delta}$ in two dimensions. Physically it can be interpreted as follows: for values $\Delta<\Delta_{\mathrm{c}}$ all of the excess is absorbed in the background fluctuations, then, at the transition point $\Delta=\Delta_{c}$, a fraction of $2 / 3$ of the excess forms a droplet while the rest of the excess remains as background fluctuations. For values $\Delta>\Delta_{c}$ the droplet grows and thereby absorbs more and more of the background fluctuations.

Numerical results. - The main aim of our work was to test from which system sizes on the theoretical results presented in the last section start to yield a good description of the data. In order to do so, $\lambda_{\Delta}$, the fraction of the excess of magnetisation in the largest droplet, had to be measured in dependence on $\Delta$. All simulations were performed at a 
TABLE I - Numerical values for the magnetisation $m_{0}$, susceptibility $\chi$ and Wulff interfacial free energy density $\tau_{\mathrm{W}}$ entering the parameters $\Delta=\Delta\left(v_{L}, m_{0}, \chi, \tau_{\mathrm{W}}\right)$ and $\theta=\theta\left(m_{0}, \chi, \tau_{\mathrm{W}}\right)$ defined in eqs. (5) and (6) at the simulation temperature $T=1.5$.

\begin{tabular}{ccccccc}
\hline$T$ & $T_{c}$ & $m_{0}$ & $\chi$ & $\tau_{\mathrm{W}}$ & $2 m_{0}^{2} / \tau_{\mathrm{W} \chi}$ & $\theta$ \\
\hline 1.500 & 2.269 & 0.9865 & 0.02708 & 4.245 & 16.93 & 0.2993 \\
\hline
\end{tabular}

temperature $T=1.5 \approx 0.66 T_{\mathrm{c}}$ which gives a good compromise between simulation speed (freezing at low temperatures) and compactness of the droplet (see the r.h.s. of fig. 3 for a typical configuration).

In order to get the correct scaling for the abscissa, $\Delta\left(v_{L}, m_{0}, \chi, \tau_{\mathrm{W}}\right)$ had to be calculated according to eq. (5). Having a constant magnetisation $M$, the value of $v_{L}$ is fixed by eq. (2). The spontaneous magnetisation $m_{0}$ is given by the famous Onsager-Yang analytic solution $[12,13]$

$$
m_{0}(\beta)=\left(1-\sinh ^{-4}(2 \beta)\right)^{1 / 8},
$$

and also the susceptibility $\chi$ is basically known to arbitrary precision from very long series expansions, e.g., Orrick et al. [14] give the formula

$$
\chi(\beta)=\beta \sum_{i=0}^{n} c_{i} u^{2 i} \quad \text { with } \quad u=\frac{1}{2 \sinh (2 \beta)}
$$

and $c=\{0,0,4,16,104,416,2224,8896,43840,175296,825648,3300480,15101920, \ldots\}$ up to order 323 (the last term contributes $\approx 0.28 \times 10^{-158}$ ). Finally, for the free energy $\Sigma_{\mathrm{W}}$ of the Wulff droplet, Leung and Zia [15] were able to derive the analytic expression $\Sigma_{\mathrm{W}}=2 \sqrt{W \Sigma}$. Here, $\Sigma$ is the volume of the droplet and $W$ is the volume bounded by the Wulff plot. Putting $\Sigma=1$ gives the interfacial free energy per unit volume

$$
\tau_{\mathrm{W}}(\beta)=2 \sqrt{W} .
$$

In the case of the Ising square lattice the volume of the Wulff plot is given by [15]

$$
W=\frac{4}{\beta^{2}} \int_{0}^{\beta \sigma_{0}} \mathrm{~d} x \cosh ^{-1}\left[\frac{\cosh ^{2}(2 \beta)}{\sinh (2 \beta)}-\cosh (x)\right],
$$

where $\sigma_{0}=2+\ln [\tanh (\beta)] / \beta$ is the interface tension of the $(1,0)$ surface $(i . e$., in direction of the axis).

Table I gives the numerical values for eqs. (8), (9) and (10) at the simulation temperature $T=1.5$. It is interesting to note that under the crude assumption of an isotropic interface tension $\sigma_{0}$, and therefore a circular Wulff shape, the interface tension per unit volume at $T=1.5$ is $2 \sqrt{\pi} \sigma_{0}=4.219$. This is quite close $(99.37 \%)$ to the correct value $\tau_{\mathrm{W}}=4.245$.

In a first step we determined the relevant region of the magnetisation roughly. This was done utilising a multimagnetic simulation and inspecting the distribution of the magnetisation shown in fig. 2 visually. The distribution exhibits for larger lattice sizes a clear cusp which divides the evaporated and condensed region. Within the evaporated region it has a Gaussian form according to eq. (3), while in the condensed region a stretched exponential behaviour is visible, cf. eq. (4). To get a feeling on what the configurations look like on the different sides of the cusp, fig. 3 displays an evaporated and condensed system, respectively. Both systems have the same magnetisation which was chosen to be that at the transition point. Even so the 


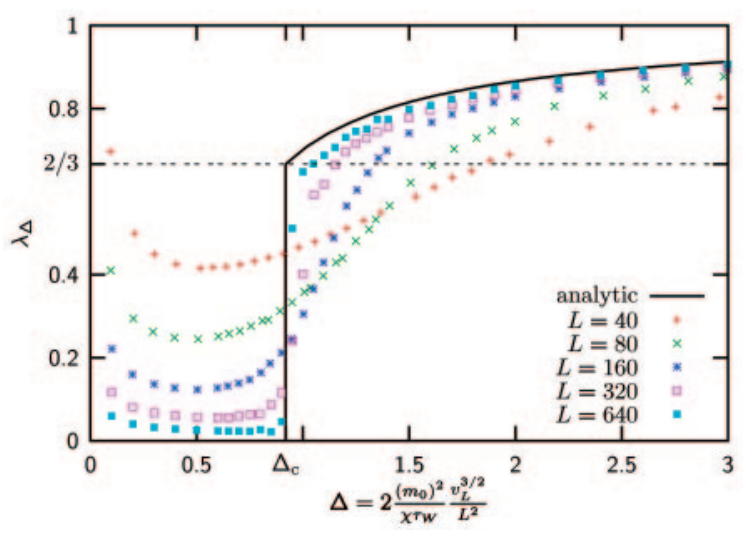

Fig. 5 - Two-dimensional Ising model with nearest-neighbour interaction on square lattices of size $L=40,80, \ldots, 640$ with periodic boundary conditions at the temperature $T=1.5 \approx 0.66 T_{c}$. The error bars are not plotted since their size is much smaller than that of the data symbols. The solid line shows the analytic solution in the limit $L \rightarrow \infty$.

pictures show situations that occurred during a simulation, they represent the extreme cases regarding the size of the largest droplet.

We compared the observed cusp region to the theoretically expected value

$$
M(\Delta)=V m_{0}-\left(\frac{2 \Delta \chi \tau_{\mathrm{W}} V}{\sqrt{2 m_{0}}}\right)^{2 / 3}=V m_{0}-\Delta^{2 / 3} \theta V^{2 / 3},
$$

obtained by inserting eq. (2) in (5) and solving for $M$. For $\Delta=0.92 \approx \Delta_{c}$ and $\theta \approx 0.2993$ from table I, this yields for $L=640$ the estimate $m \approx 0.9827$. Encouraged by the good agreement with our data in fig. 2 , we decided to fix the magnetisation by selecting a set of 38 reasonable values $\Delta_{i}=\{0.00,0.10, \ldots\}$ with an emphasis on the vicinity of $\Delta_{c}$. The corresponding values of the magnetisation must be rounded to the next allowed integer value and then the true $\Delta_{i}$ are calculated backwards.

Next, we started 38 simulations with constant magnetisation (micromagnetic). The constraint on the magnetisation was enforced utilising a Metropolis update with Kawasaki dynamics essentially exchanging a pair of unaligned spins. After every sweep a cluster decomposition was performed using the Hoshen-Kopelman [16] algorithm and the volume of the largest droplet was measured yielding the desired $\lambda_{\Delta}$. Two things are to be noted here: First, the range of magnetisations that was chosen guaranteed that the droplet was always the second largest cluster (the background is the largest cluster). Second, in the present context the volume of the cluster includes overturned spins within the cluster. Our simulations are sensitive to a degree that the proper counting of the cluster size turned out to be indeed crucial. Technically, this was handled by a so-called "flood-fill" routine [17] that ran after the Hoshen-Kopelman algorithm. In essence, it starts from an inside spin and stops when a spin that belongs to the background is reached. Very rarely ambiguous cases can occur which can be detected automatically and were taken care of by inspection. We repeated the whole procedure for five different lattices sizes $L=40,80,160,320$, and 640. Every simulation ran 20000 sweeps for the thermalisation and 200000 sweeps for measurements. To obtain the error bars reliably, 10 independent simulations were run for each data point.

Our main result, the fraction $\lambda_{\Delta}$ for various lattice sizes, is shown in fig. 5 . The solid line represents the analytical value (obtained by the minimisation of $\phi_{\Delta}(\lambda)$ in eq. (7)). For 
larger lattice sizes the theoretical value is approached by the results of the simulation. The jump from $\lambda_{\Delta} \approx 0$ to $\lambda_{\Delta} \approx 2 / 3$ at $\Delta_{\mathrm{c}} \approx 0.92$ confirms the theoretical prediction that at the evaporation/condensation transition only $2 / 3$ of the excess of the magnetisation goes into the droplet while the rest remains in the background fluctuations.

The increase of $\lambda_{\Delta}$ for $\Delta \rightarrow 0$ can be explained by the fact that the minimal cluster size is 1 and not an arbitrarily small fraction. In contrast, the excess that can be fixed analytically using eq. (5) can be much smaller than 1.

Conclusion. - Our Monte Carlo data clearly confirm the theoretical considerations of Biskup et al. $[5,6]$ and extend their exact results for very large systems to practically accessible system sizes. The observed finite-size scaling behaviour fits perfectly with their predictions for the infinite system. All simulations were performed in thermal equilibrium and the abundance of droplets of intermediate size could be confirmed. At the moment, additional simulations for different models are performed that should prove the universal aspects of the theory.

$$
* * *
$$

We are indebted to K. BINDER for sharing his physical insight into the droplet nucleation mechanism, and wish to thank R. KотECKÝ for helpful discussions on the formulation used in the present work. Work supported by the Graduiertenkolleg "Quantenfeldtheorie: Mathematische Struktur und Anwendungen in der Elementarteilchen- und Festkörperphysik" and by the Deutsche Forschungsgemeinschaft (DFG) under grant No. JA483/22-1. TN thanks the NTZ for financial support and hospitality during extended visits of the University of Leipzig.

\section{REFERENCES}

[1] Fisher M. E., Rep. Prog. Phys., 30 (1967) 615.

[2] Binder K. and Kalos M. H., J. Stat. Phys., 22 (1980) 363.

[3] Furukawa H. and Binder K., Phys. Rev. A, 26 (1982) 556.

[4] Neuhaus T. and Hager J. S., J. Stat. Phys., 113 (2003) 47.

[5] Biskup M., Chayes L. and Kotecký R., Europhys. Lett., 60 (2002) 21.

[6] Biskup M., Chayes L. and Kotecký R., Commun. Math. Phys., 242 (2003) 137.

[7] Binder K., Physica A, 319 (2003) 99.

[8] Virnau P., MacDowell L. G., Müller M. and Binder K., Computer Simulation Studies in Condensed Matter Physics XVI, edited by Landau D. P., Lewis S. M. and Schüttler H.-B. (Springer, Berlin) 2004.

[9] Biskup M., Chayes L. and Kotecký R., J. Stat. Phys., 116 (2003) 175.

[10] Dobrushin R. L., KoteckÝ R. and Shlosman S. B., Wulff Construction. A Global Shape from Local Interaction (American Mathematical Society, Providence, RI) 1992; J. Stat. Phys., $\mathbf{7 2}(1993) 1$.

[11] WulfF G., Z. Kristallogr. Mineral., 34 (1901) 449.

[12] Onsager L., Nuovo Cimento (Suppl.), 6 (1949) 261.

[13] Yang C. N., Phys. Rev., 85 (1952) 808.

[14] Orrick W. P., Nickel B. G., Guttmann A. J. and Perk J. H. H., Phys. Rev. Lett., 86 (2001) 4120.

[15] Leung K. and Zia R. K. P., J. Phys. A, 23 (1990) 4593.

[16] Hoshen J. and Kopelman R., Phys. Rev. B, 14 (1976) 3438.

[17] Agoston M. K., Computer Graphics and Geometric Modelling (Springer, London) 2004. 